Pacific Journal of Mathematics

TUBULAR NEIGHBORHOODS OF HILBERT CUBE 


\title{
TUBULAR NEIGHBORHOODS OF HILBERT CUBE MANIFOLDS
}

\author{
W. O. NOWELL, JR. ${ }^{1}$
}

Let $M$ and $N$ be $Q$-manifolds and let $i$ be a locally flat embedding of $N$ into $M$. It is shown that if $N=Q \times R^{n}$, then $i$ must be flat. The following version of the KirbySiebenmann codimension 2 tubular neighborhood theorem is proved. If $i$ is locally flat of codimension 2 , then the embedded submanifold has a tubular neighborhood, and any two such tubular neighborhoods are isotopic. Among the tools developed is a relative version of $Z$-set unknotting.

1. Introduction. An open neighborhood $E$ of $N$ in $M$ is said to be a tubular neighborhood (of codimension $n$ ) if there is a retraction $p: E \rightarrow N$ such that $(E, p, N)$ is a fiber bundle with fiber Euclidean $n$-space $R^{n}$ and 0 -section $N$. That is, there exists an open covering $\left\{U_{i}\right\}$ of $B$ and, for each index $i$, a homeomorphism $\varphi_{i}: U_{i} \times R^{n} \rightarrow p^{-1}\left(U_{i}\right)$ such that $p \varphi_{i}(x, y)=x$ for all $x \in U_{i}$, and, furthermore for any $i$ and any $x \in U_{i}, \varphi_{i}(x, 0)=x$.

The following is a closely related concept. A closed embedding $i: N \rightarrow M$ is said to be locally flat (of codimension $n$ ) if for each $x_{0} \in N$ there is an open neighborhood $U$ of $x_{0}$ in $N$ and an open embedding $h: U \times R^{n} \rightarrow M$ such that $h(x, 0)=i(x)$ for all $x \in U$. If $U$ can be taken to be all of $N$, then the embedding is said to be flat. It is an immediate consequence of the definition that if $N$ has a tubular neighborhood in $M$, then the inclusion $N \rightarrow M$ is locally flat. Thus, for example, a wild knot in $R^{3}$ cannot have a tubular neighborhood. (For a discussion of local flatness and tameness, see also [1].)

In case $M$ and $N$ are differentiable manifolds there are naturally defined notions of tangent and normal vector bundles, and most questions can be answered in terms of these structures. In particular, every smooth embedding is locally flat, and in the smooth category every submanifold has a tubular neighborhood.

The problem is somewhat more difficult in the case of finite dimensional topological manifolds. Not all locally flatly embedded manifolds need have tubular neighborhoods, as is demonstrated by the counterexamples by Rourke and Sanderson [18] and by Hirsch [9]. The following results are known for $M$ and $N$ finite dimen-

1 These results first appeared in the author's doctoral dissertation at the University of Kentucky under the direction of Professor T. A. Chapman. The author is grateful to Professor Chapman for his assistance throughout the progress of the work. 
sional manifolds and $N \rightarrow M$ locally flat of codimension $n$. The collaring theorem of Brown [1] implies that if $n=1$, then $N$ has a tubular neighborhood. Kirby and Siebenmann [12] have shown that if $n=2$ and $\operatorname{dim} N \neq 2$, then $N$ has a tubular neighborhood. Milnor [17] proved the stable existence of tubular neighborhoods, that is, if the integer $k$ is sufficiently large, then $N \times\{0\}$ has a tubular neighborhood in $M \times R^{k}$. Stern [19] has proved a stronger result for large codimension, namely, if $n \geqq \operatorname{dim} N-j-1$ and $n \geqq 5+j$, where $j=0,1,2$, then $N$ has a tubular neighborhood.

The purpose of this paper is to investigate similar questions where $M$ and $N$ are $Q$-manifolds, that is, manifolds modeled on the Hilbert cube $Q$. Here is our first main result.

Theorem 1. Let $M$ be a Q-manifold and let $m \geqq 0$. If $i: Q \times$ $R^{m} \rightarrow M$ is locally flat, then $i$ is flat.

The case $m=0$ has been proved previously by Chapman [3]. The answer to the following is not known.

Question. If " $Q \times R^{m}$ " is replaced by "a contractible $Q$-manifold $N$ " in the statement of Theorem 1 , is the theorem still true?

Brown's theorem applies to $Q$-manifolds, so it is known that if $N \rightarrow M$ is locally fiat of codimension 1 , then $N$ has a tubular neighborhood. Our second main result is a $Q$-manifold version of the Kirby-Siebenmann codimension 2 theorem.

THEOREM 2. If $(M, N)$ is a Q-manifold pair and the inclusion $N \rightarrow M$ is locally flat of codimension 2, then $N$ has a tubular neighborhood. Furthermore any two such tubular neighborhoods are isotopic as normal microbundles.

The uniqueness part of this statement means that if $p_{i}: E_{i} \rightarrow N$, $i=1,2$, define the tubular neighborhoods, then there is a homeomorphism $h: M \rightarrow M$ such that $h=i d$ on $N, h$ is isotopic to $i d$ rel $N$, and there exists some open neighborhood $U$ of $N$ in $E_{1}$ such that $h(U) \subset E_{2}$ and $p_{2} h=p_{1}$ on $U$.

We note in conclusion that Chapman [4] has recently proved that there exists a codimension 3 locally flat embedding $N \hookrightarrow M$ of a $Q$-manifold such that $N$ has no tubular neighborhood and, moreover, no stabilization $N \times\{0\} \rightarrow M \times R^{k}$ has a tubular neighborhood.

The paper is organized as follows. Section 2 consists of preliminaries including a summary of the basic results from $Q$-manifold theory and bundle theory which will be used subsequently. Section 
3 develops some new $Q$-manifold results. In particular Theorem 3.2 is a relative version of the notion of $Z$-set unknotting, a concept introduced by R. D. Anderson in the study of the Hilbert cube and one which has been central to the study of $Q$-manifolds. This particular theorem will be used in the proof of Theorem 1.

The main technical aspects of Theorems 1 and 2 are proved in $\S 4$. The proofs of the theorems themselves are then contained in $\S \S 5$ and 6 respectively.

2. Preliminaries. All spaces are locally compact, separable and metric unless otherwise specified. We use the standard notation $R^{n}, B^{n}$ and $S^{n-1}$ to mean $n$-dimensional Euclidean space, the unit $n$-ball and the unit $(n-1)$-sphere respectively. We also use $\|x\|$ to denote the Euclidean norm of a point $x$.

If $f, g: X \rightarrow Y$ are maps and $\alpha$ is an open cover of $Y$, then we say that $f$ is $\alpha$-close to $g$ provided that for each $x \in X$ there is some $U \in \alpha$ such that both $f(x)$ and $g(x)$ are elements of $U$. We say that a homotopy $F_{t}: f \stackrel{\alpha}{\simeq} g$ is an $\alpha$-homotopy and that $f$ and $g$ are $\alpha$ homotopic (denoted $f \simeq g$ ) provided that for each $x \in X$ there is some $U_{x} \in \alpha$ such that $\left\{F_{t}(x): 0 \leqq t \leqq 1\right\} \subset U_{x}$. A proper map is a map for which inverse images of compact sets are compact. Such notions as proper homotopy and proper homotopy equivalence are defined in anology with the corresponding notions from the ordinary homotopy category. A proper map $f: X \rightarrow Y$ is an $\alpha$-equivalence if there is a proper homotopy inverse $g: Y \rightarrow X$ so that $f g$ is $\alpha$ homotopic to $i d_{Y}$ and $g f$ is $f^{-1}(\alpha)$-homotopic to $i d_{X}$, where $f^{-1}(\alpha)=$ $\left\{f^{-1}(U)=U \in \alpha\right\}$.

We represent the Hilbert cube $Q$ as the countably infinite product of closed intervals $[-1,1]$. A space $M$ is a $Q$-manifold if it has a basis consisting of elements which are homeomorphic to open subsets of $Q$. Many $Q$-manifold results involve the notion of a $Z$-set. A closed set $A$ in a space $X$ is a $Z$-set in $X$ if, given any open cover $\alpha$ of $X$, there is a map $f: X \rightarrow X-A$ such that $f$ is $\alpha$-close to $i d_{X}$. An embedding $f: X \rightarrow Y$ is a $Z$-embedding if $f(X)$ is a $Z$-set in $Y$.

Our basic reference for $Q$-manifold theory is [2], which should be consulted by a reader interested in more details. For convenience we list some of the basic machinery which will be used frequently in the sequel.

2.1. $Z$-set unknotting [2, Theorem 19.4].

2.2. Approximation by $Z$-embedding [2, Theorem 18.2].

2.3. Stability of $Q$-manifolds [2, Theorem 15.1].

2.4. Triangulation of $Q$-manifolds [2, Theorem 37.2].

2.5. Edwards' ANR theorem [2, Theorem 44.1]. 
We will also freely use the terminology associated with fiber bundles in standard references such as Husemoller [11] and the notion of microbundle as defined by Milnor [16]. Related concepts concerning microbundles may be found in [15].

If $M$ is an $m$-dimensional topological manifold or a $Q$-manifold, and $N$ is a submanifold of $M$, then a normal microbundle for $N$ in $M$ consists of an open neighborhood $E$ of $N$ and a retraction $p: E \rightarrow N$ so that $N \hookrightarrow E \stackrel{p}{\rightarrow} N$ is a microbundle. Thus every tubular neighborhood is a normal microbundle. As a consequence of the Kister-Mazur theorem [13], every normal microbundle contains a tubular neighborhood. Thus the notions are equivalent so long as we are free to pass to a smaller neighborhood. (The versions of the Kister-Mazur theorem proved by Kister [13] and by KuiperLashof [15] include the hypothesis that the base space is a polyhedron. We need the base space to be a $Q$-manifold. Such a result is readily proved from Kister's result, and, in any case, the generalized version of the theorem proved by Holm [10] is certainly broad enough for our purposes.)

3. $Z$-sets in a locally flatly embedded $Q$-manifold. The purpose of this section is to establish Theorem 3.2, a relative version of $Z$-set unknotting. That is, we show that if $N$ is a locally flatly embedded submanifold of a $Q$-manifold $M$, then $Z$-set unknotting in $N$ can be extended to a homeomorphism of $M$ which preserves $N$.

Lemma 3.1 is a technical tool used in the proof of Theorem 3.2. It will also be used in its own right in the sequel.

Lemma 3.1. Let $M$ and $N$ be Q-manifolds, where $N$ is a locally flat submanifold of codimension $n$, and let $\varphi$ be any homeomorphism of $N$ onto $N \times I$. Then there exists a homeomorphism $h:(M, N) \rightarrow$ $(M, N)$ such that $h \varphi^{-1}(x, 1 / 3)=\varphi^{-1}(x, 2 / 3)$ for all $x \in N$.

Furthermore, if $N$ is compact and if $W$ is any open neighborhood of $\varphi^{-1}(N \times[1 / 3,2 / 3])$ in $M$, then $h$ can be chosen so that its support is a compact subset of $W$.

Proof. In order to simplify notation, we identify $N$ and $\varphi(N)$ and thus regard $N \times I$ as a locally flat submanifold of $M$.

Case 1. $N$ is compact. It follows from elementary compactness arguments and the locally flat structure there exist open sets $U_{i}$ and $V_{i}$ of $N, i=1, \cdots, m$, and open intervals $\left(a_{j}, b_{j}\right), j=1, \cdots, k$, satisfying the following properties.

(1) $\left\{U_{i}\right\}_{i=1}^{m}$ is an open cover of $N$.

(2) $\bar{U}_{i} \subset V_{i}$ for each $i$. 
(3) $0<a_{1}<\cdots<a_{k}<2 / 3$ and $1 / 3<b_{1}<\cdots<b_{k}<1$.

(4) $\left\{\left(a_{j}, b_{j}\right)\right\}_{j=1}^{k}$ covers $[1 / 3,2 / 3]$.

(5) $N \times\left(a_{1}, b_{k}\right) \subset W$.

(6) For each pair $(i, j)$ of indices, there is an open embedding

$$
g_{i, j}: V_{i} \times\left(a_{j}, b_{j}\right) \times R^{n} \longrightarrow M
$$

with $g_{i, j}(x, 0)=x$ for all $x \in V_{i} \times\left(a_{j}, b_{j}\right)$.

We choose numbers $t_{0}=1 / 3<t_{i}<\cdots<t_{k}=2 / 3$ so that

$$
t_{j} \in\left(a_{j}, b_{j}\right) \cap\left(a_{j+1}, b_{j+1}\right), \quad j=1, \cdots, k-1 .
$$

For any fixed choice of $i$ and for each choice of $j$ from 1 to $k$, it follows from a standard Tietze extension argument that there is a homeomorphism

$$
\theta=V_{i} \times\left(a_{j}, b_{j}\right) \longrightarrow V_{i} \times\left(a_{j}, b_{j}\right)
$$

which has compact support and is such that $\theta\left(x, t_{j-1}\right)=\left(x, t_{j}\right)$ for all $x \in U_{\imath}$. The geometry is illustrated by the following picture.

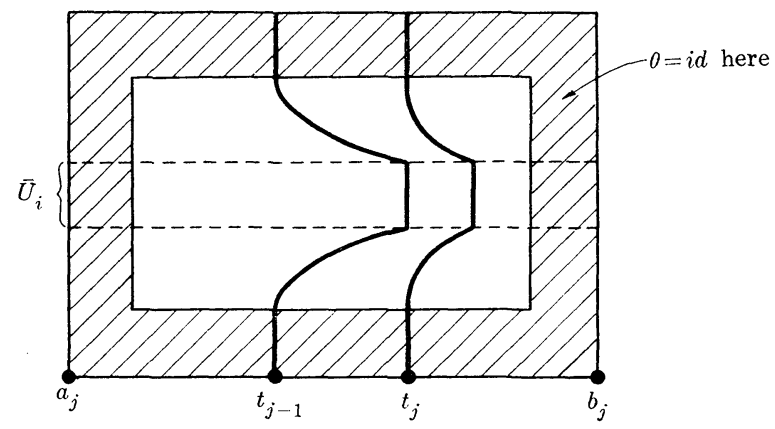

It is, moreover, clear that $\theta$ can be extended to a homeomorphism on $g_{i, j}\left(V_{i} \times\left(a_{j}, b_{j}\right) \times R^{n}\right)$ whose support is a compact subset of $W$. Thus, extending by the identity, we obtain a homeomorphism of pairs $f_{i, j}:(M, N \times I) \rightarrow(M, N \times I)$ such that $f_{i, j}\left(x, t_{j-1}\right)=\left(x, t_{j}\right)$ for all $x \in U_{i}$. Therefore the homeomorphism

$$
f_{i}=f_{i, k} \circ f_{i, k-1} \circ \cdots \circ f_{i, 1}:(M, N \times I) \longrightarrow(M, N \times I)
$$

has the property that $f_{i}(x, 1 / 3)=(x, 2 / 3)$ for all $x \in U_{i}$.

Now we construct $h$ by piecing together the $f_{i}$. That is, we construct a finite sequence of homeomorphisms $h_{1}=f_{1}, h_{2}, \cdots, h_{m}=h$ of $(M, N \times I)$ onto itself such that $h_{i}(x, 1 / 3)=(x, 2 / 3)$ for all $x \in$ $U_{1} \cup \cdots \cup U_{i}$ and such that $p_{1} h_{i}(x, t)=x$ for all $(x, t) \in N \times I$, where $p_{1}$ is projection on the first coordinate of $N \times I$.

We note that $h_{1}=f_{1}$ has the desired properties. The following picture illustrates how $h_{i+1}$ is constructed from $h_{i}$. 


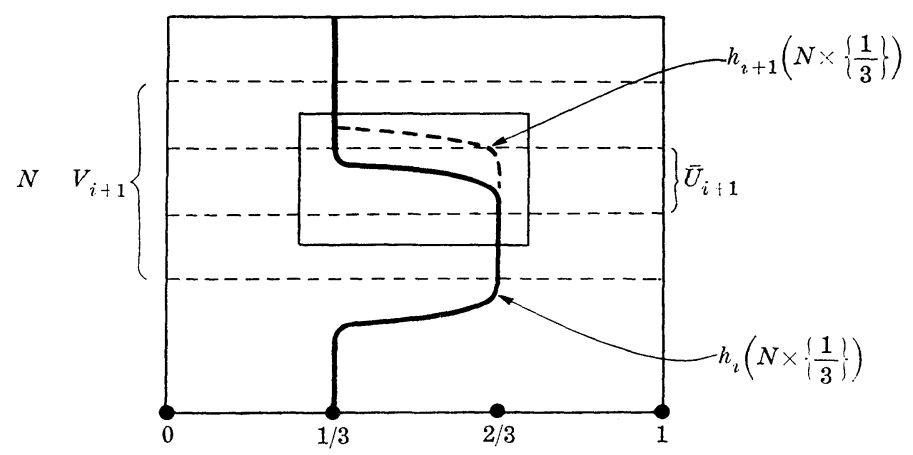

The details of the formal construction are again routine and are left to the reader.

Case 2. $N$ is locally compact. Represent $N$ as the union of two closed subsets $A$ and $B$, where $A=\bigcup_{i=1}^{\infty} A_{i}, B=\bigcup_{i=1}^{\infty} B_{i}$ and $\left\{A_{i}\right\}$ and $\left\{B_{i}\right\}$ are collections of pairwise disjoint compact subsets of $N$. Applying Case 1 to each of the $A_{i}$ separately, we obtain a homeomorphism $f:(M, N \times I) \rightarrow(M, N \times I)$ so that $f(x, 1 / 3)=(x, 2 / 3)$ for all $x \in A$. Then we can apply the same techniques as before to define a family of homeomorphisms $g_{i}$ so that $g_{i} f(x, 1 / 3)=(x, 2 / 3)$ for all $x \in A \cup B_{i}$. Moreover, since the $B_{i}$ are disjoint, the $g_{i}$ can be chosen with disjoint supports, so the infinite composition is a well defined homeomorphism $g$. Therefore, $h=g f$ is the desired homeomorphism.

THEOREM 3.2. Let $M$ and $N$ be Q-manifolds with the inclusion $i: N \rightarrow M$ a locally flat embedding, and let $A$ and $B$ be Z-sets in $N$. If $f: A \rightarrow B$ is a homeomorphism which is properly homotopic to the identity in $N$, then there is a homeomorphism $h:(M, N) \rightarrow(M, N)$ with $h \mid A=f$.

Proof. Case 1. $A \cap B=\phi$. It is an elementary exercise to show that there is a homeomorphism $\varphi: N \rightarrow N \times I$ such that $\varphi(B) \subset N \times$ $\{2 / 3\}$. Note that $\varphi(A) \cap \varphi(B)=\phi$, so we can define a $Z$-embedding $g: \varphi(A) \cup \varphi(B) \rightarrow N \times I$ by $g \varphi(x)=\varphi(x)$ if $x \in B$ and, if $x \in A, g \varphi(x)=$ $(y, 1 / 3)$ where $\varphi f(x)=(y, 2 / 3)$. Now let $F: A \times I \rightarrow N$ be a proper homotopy such that $F_{0}=i d$ and $F_{1}=f$. Note that $\varphi F$ is then a proper homotopy between $\varphi$ and $\varphi f$, and, furthermore $\varphi f \simeq g \varphi \mid A$ by a straight line homotopy. Thus $g \mid \varphi(A) \simeq i d$ and $g \mid \varphi(B)=i d$, so by $Z$-set unknotting in $N \times I$ there is a homeomorphism extending $g$ to $N \times I$. That is, there exists a homeomorphism $\psi: N \rightarrow$ $N \times I$ such that $\psi(A) \subset N \times\{1 / 3\}$ and $\psi f \psi^{-1}(x, 1 / 3)=(x, 2 / 3)$ whenever $(x, 1 / 3) \in \psi(A)$. 
Then by Lemma 3.1 there is a homeomorphism $h:(M, N) \rightarrow$ $(M, N)$ such that $h \psi^{-1}(x, 1 / 3)=\psi^{-1}(x, 2 / 3)$ for any $x \in N$. In particular, if $y \in A$, then $\psi(y)=(x, 1 / 3)$ and we have $h(y)=\psi^{-1}(x, 2 / 3)=$ $f \psi^{-1}(x, 1 / 3)=f(y)$ as required.

Case 2. $A \cap B \neq \varnothing$. Let $\varphi: N \rightarrow N \times I$ be a homeomorphism such that $\varphi(A \cup B) \subset N \times\{1 / 3\}$. Then by Lemma 3.1 there is a homeomorphism $h_{1}:(M, N) \rightarrow(M, N)$ such that $\varphi h_{1}(B) \subset N \times\{2 / 3\}$. In particular $A \cap h_{1}(B)=\varnothing$. Then it follows from Case 1 that the homeomorphism $h_{1} f: A \rightarrow h_{1}(B)$ can be extended to $h_{2}:(M, N) \rightarrow(M, N)$. Therefore $h=h_{1}^{-1} h_{2}$ is the required homeomorphism.

REMARK. The reader may observe that our Theorem 3.2 makes no mention of the cover control frequently associated with $Z$-set unknotting. The theorem as stated is sufficient for our later use, but a full analogy of Theorem 19.4 of [2] complete with cover control can be proved by imitating the techniques used in Chapter IV of [2] and slightly modifying the above proofs.

4. Two meshing lemmas. In this section we develop the main technical results which will be used in the proofs of Therem 1 and Theorem 2. Lemma 4.3 will be used to mesh trivial microbundles of arbitrary codimension and Lemma 4.7 will be used to mesh arbitrary microbundles of codimension 2 .

The proofs of these lemmas will both rely on the notion of a pinched tube. We now define this construction for a trivial tube. A similar construction for a codimension 2 tube will be given later.

Let $(M, N)$ be a $Q$-manifold pair. Suppose $N$ is homeomorphic to $Q \times R^{m}$ for some $m \geqq 1$. A trivial tubular neighborhood for such a manifold $N$ is then defined by a homeomorphism

$$
f:\left(Q \times R^{m} \times R^{n}, Q \times R^{m} \times\{0\}\right) \longrightarrow(D, N),
$$

where $D$ is an open neighborhood of $N$ in $M$. Then for any choice of a map $r: 2 \dot{B}^{m} \rightarrow(0, \infty)$ such that

(1) $r(x) \leqq r(0)$ for all $x \in 2 \dot{B}^{m}$,

(2) $r(x)=r(0)$ for all $x \in B^{m}$, and

(3) $\lim _{\|: x \mid \rightarrow \rightarrow 2} r(x)=0$,

a closed tube can be constructed as follows. Let

$$
E=\left\{f(q, x, y) \in D: x \in 2 \stackrel{\circ}{B}^{m},\|y\| \leqq r(x)\right\},
$$

let $B=f\left(Q \times 2 \dot{B}^{m} \times\{0\}\right)$ and let $p: E \rightarrow B$ be the retraction defined by $p f(q, x, y)=f(q, x, 0)$.

We shall call $(E, p, B)$ the pinched tube determined by the 
embedding $f$ and the map $r$. We shall also refer to $r$ as the pinching function for the tube and call $A=\{f(q, x, y) \in E:\|y\|=r(x)\}$ the boundary of the pinched tube. The following picture illustrates this notion.

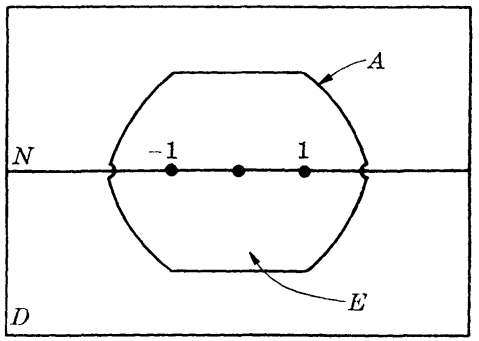

The following observations are immediate consequences of the definition and will be used subsequently without further comment.

Proposition 4.1. Let $(E, p, B)$ be a pinched tube constructed from a trivial tube as described above.

(1) Given any neighborhood $U$ of $B$ in $M$, there is a choice of pinching function $r$ so that $E \subset U$.

(2) For any such pinched tube there is a homeomorphism $g: B \times B^{n} \rightarrow E$ such that $g(x, 0)=x$ and $p g(x, y)=x$ for all $x \in B$. Moreover, $g \mid B \times S^{n-1}$ is a homeomorphism onto $A$.

(3) For any such pinched tube there is a radially defined retraction $\rho: f\left(Q \times 2 \dot{B}^{m} \times R^{n}\right)-B \rightarrow A$ such that $p \rho=p$ on $f(Q \times$ $\left.2 \dot{B}^{m} \times R^{n}\right)-B$.

We will also need the following recent result by Chapman.

THEOREM 4.2 ([5, Theorem 1]). For each open cover $\alpha$ of a $Q$ manifold $B$ there is an open cover $\beta$ of $B$ so that if $p: E \rightarrow B$ is a fiber bundle, with fiber a compact ANR for which $\pi_{1}$ of each component is free or free abelian, then any $p^{-1}(\beta)$-equivalence from a $Q$-manifold $M$ to $E$ is $p^{-1}(\alpha)$-homotopic to a homeomorphism.

LEMMA 4.3. If $f: Q \times R^{m} \times R^{n} \rightarrow Q \times R^{m} \times R^{n}$ is an open embedding such that $f=i d$ on $Q \times R^{m} \times\{0\}$, then there exists $a$ homeomorphism $h: Q \times R^{m} \times R^{n} \rightarrow Q \times R^{m} \times R^{n}$ such that

(1) h has compact support,

(2) $h=i d$ on $Q \times R^{m} \times\{0\}$, and

(3) for some $\varepsilon>0$, either fh $=i d$ or fh $=i d \times$ a on $Q \times B^{m} \times$ $\varepsilon B^{n}$, where $a$ is the standard orientation reversing map $a\left(x_{1}, x_{2}\right.$, $\left.\cdots, x_{n}\right)=\left(-x_{1}, x_{2}, \cdots, x_{n}\right)$. 
Proof. The proof depends on consideration of three pinched tubes. In accordance with the notation introduced above let $B=Q \times 2 B^{m} \times\{0\}$ and $D=f\left(Q \times R^{m} \times R^{n}\right)$. The pinched tubes $\left(E_{1}, p_{1}, B\right)$ and $\left(E_{3}, p_{3}, B\right)$ are determined by the identity embedding and pinching functions $r_{1}$ and $r_{3}$. The last pinched tube, $\left(E_{2}, p_{2}, B\right)$ is determined by $f$ and a pinching function $r_{2}$ chosen so that $r_{2}(x)<r_{1}(x)$ for all $x \in 2 \dot{B}^{m}$. Moreover, the $r_{i}$ are chosen so that $E_{1} \subset D, E_{2} \subset{\stackrel{\circ}{E_{1}}}_{1}$ and $E_{3} \subset{\stackrel{\circ}{E_{2}}}_{\text {. }}$. Here is a picture.

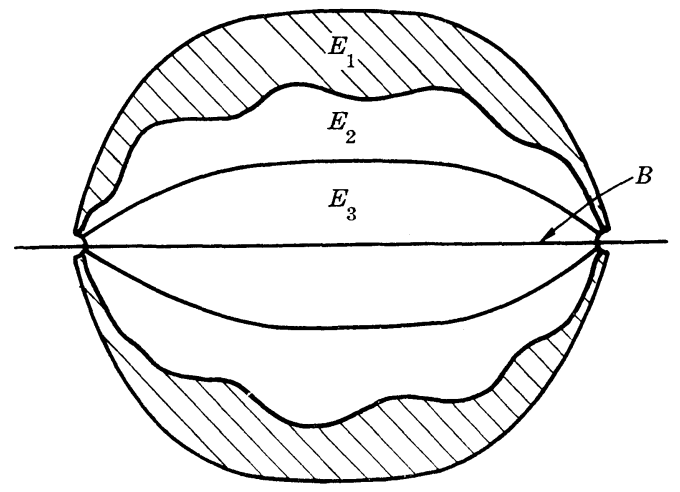

Denote by $A_{k}$ the boundaries of $E_{k}, k=1,2,3$. Let $X=E_{1}-\stackrel{\circ}{2}_{2}$. ( $X$ is the shaded region of the picture.) Let $D^{\prime}=f\left(Q \times 2 \dot{B}^{m} \times R^{n}\right)$ and let $p: D^{\prime} \rightarrow B$ be the natural retraction defined by $p f(q, x, y)=$ $f(q, x, 0)=(q, x, 0)$.

Assertion 1. The inclusion $i: A_{2} \hookrightarrow X$ is a proper homotopy equivalence. Moreover, if $\alpha$ is an open cover of $B$, then the pinched tubes can be chosen so that $i$ has a homotopy inverse $u: X \rightarrow A_{2}$ which is a $p^{-1}(\alpha)$-equivalence.

We prove the assertion by defining $u$ directly. Let $\rho: D^{\prime}-B \rightarrow A_{2}$ be the natural fiber preserving retraction and let $\sigma: E_{1} \rightarrow E_{3}$ be the natural fiber preserving homeomorphism which fixes $B$ and which carries $A_{1}$ to $A_{3}$. (See Proposition 4.1.) Then $u=\rho \sigma$ is the desired homotopy inverse.

Let $\sigma_{t}: E_{1} \rightarrow E_{1}$ be a fiber preserving homotopy such that $\sigma_{0}=i d$ and $\sigma_{1}=\sigma$. Define a retraction $\rho_{1}: E_{1}-B \rightarrow X$ by $\rho_{1}=\rho$ on $E_{2}-B$ and $\rho_{1}=i d$ on $E_{1}-E_{2}$. Note that $\rho \sigma_{t}: i d_{A_{2}} \simeq u i$ in $A_{2}$ and $\rho_{1} \sigma_{t}$ : $i d_{X} \simeq i u$ in $X$. It is routine to show that $\rho \sigma_{t}$ is a $p^{-1}(\alpha)$-homotopy and that $\rho_{1} \sigma_{t}$ is a $(p u)^{-1}(\alpha)$-homotopy provided that the values of the values of the pinching functions are chosen sufficiently close to 0 . The deails are left to the reader.

Assertion 2. If $\alpha$ is any open cover of $B$, then the pinched 
tubes can be chosen so that there exists a homeomorphism $h_{1}: E_{1} \rightarrow E_{1}$ such that

(1) $h_{1}=f^{-1}$ on $E_{2}$ and

(2) $h_{1}$ is $p^{-1}(\alpha)$-close to the identity.

Proof. In view of Assertion 1, we apply Theorem 4.2. That is, for any open cover $\beta$ of $B$ the pinched tubes can be chosen so that there exists a homeomorphism $g: X \rightarrow A_{2}$ which is $p^{-1}(\alpha)$ homotopic to the previously defined map $u$. It then follows from the stability of $Q$-manifolds that there is a homeomorphism $\bar{g}: X \rightarrow$ $A_{2} \times I$ such that $\bar{g}$ is $\pi^{-1}(\beta)$-homotopic to the embedding $u \times 0$, where $\pi$ is defined by $\pi(x, t)=p(x)$.

We will now use $Z$-set unknotting to modify $\bar{g}$ so that $\bar{g} \mid A_{2}=$ $i d \times 0$. All that is needed is to recall from the proof of Assertion 1 that we may require $u \mid A_{2}$ to be $p^{-1}(\beta)$-homotopic to the identity on $A_{2}$. Thus $\bar{g} \simeq u \times 0 \simeq i d \times 0$ on $A_{2}$ by homotopies with arbitrarily fine horizontal control. Therefore by controlled $Z$-set unknotting there is a correcting homeomorphism having the same cover control.

Now the proof of the assertion follows quickly. The pinching function $r_{2}$ was chosen so that $f^{-1}\left(E_{2}\right) \subset \mathscr{E}_{1}^{\prime}$. Moreover, it is clear that the homeomorphism indicated in Proposition 4.1(2) can be used to construct a homeomorphism $\psi: f^{-1}\left(A_{2}\right) \times I \rightarrow E_{1}-f^{-1}\left(\dot{E}_{2}\right)$ which is fiber preserving (with respect to projection $p_{1}$ ) and such that $\psi(x, 0)=x$. Thus we construct $h_{1}$ as follows.

$$
h_{1}: X \stackrel{\bar{g}}{\longrightarrow} A_{2} \times I \stackrel{f^{-1} \times i d}{\longrightarrow} f^{-1}\left(A_{2}\right) \times I \stackrel{\psi}{\longrightarrow} E_{1}-f^{-1}\left(\dot{E}_{2}^{\circ}\right) .
$$

Now extend $h_{1}$ to all of $E_{1}$ by means of $f^{-1}$.

It is clear that we can require that $p h_{1}$ be $\alpha$-close to $p$ by requiring that the cover $\beta$ be sufficiently fine and that the values of the pinching functions be chosen sufficiently small.

We are now ready to complete the proof of the lemma.

Since $E_{1}$ is naturally homeomorphic to $B \times B^{n}, h_{1}$ induces a homeomorphism $\widetilde{h}: B \times B^{n} \rightarrow B \times B^{n}$ such that $\widetilde{h}=i d$ on $B \times\{0\}$. Define a map $\varphi: S^{n-1} \rightarrow S^{n-1}$ by $\varphi(x)=r \pi_{2} \widetilde{h}\left(b_{0}, x\right)$ where $b_{0} \in B, \pi_{2}$ is projection onto $B^{n}$, and $r$ is radial retraction. Since $B$ is contractible, $\varphi$ is a homotopy equivalence. Therefore $\varphi$ is homotopic to either the identity or the orientation reversing map on $S^{n-1}$.

Define a map $\tilde{\eta}: B \times S^{n-1} \rightarrow B \times S^{n-1}$ by $\tilde{\eta}(b, x)=\left(\pi_{1} \widetilde{h}(b, x), \varphi(x)\right)$ where $\pi_{1}$ is projection onto $B$. Clearly $\tilde{\eta}$ is fiber homotopic to $\tilde{h}$ in $B \times\left(B^{n}-\{0\}\right)$. Moreover, since $h_{1}$ has been chosen so that $\pi_{1} \tilde{h}$ is close to $\pi_{1}$, it follows easily from well known properties of ANRs that $\pi_{1} \tilde{h} \simeq \pi_{1}$ by means of a small homotopy. Therefore either $\tilde{\eta} \simeq i d$ or 
$\tilde{\eta} \simeq i d \times a$, and, in either case, the homotopy has arbitrarily fine horizontal control.

Since $\tilde{\eta}$ induces a similar map $\eta: A_{1} \rightarrow A_{1}$, the same argument shows that either $h_{1} \mid A_{1} \simeq i d$ or $h_{1} \mid A_{1} \simeq i d \times a$ in $E_{1}-E_{2}$ by a homotopy with fine horizontal control. Thus we are able to use controlled $Z$-set unknotting to modify $h_{1}$ rel $E_{2}$.

Therefore if $\alpha$ is an open cover of $B$, then the pinched tubes can be chosen so that there is a homeomorphism $h_{2}: E_{1} \rightarrow E_{1}$ such that

(1) $h_{2}=f^{-1}$ on $E_{2}$,

(2) either $h_{2}=i d$ or $h_{2}=i d \times a$ on $A_{1}$, and

(3) $h_{2}$ is $p^{-1}(\alpha)$-close to the identity.

If $h_{2}=i d$ on $A_{1}$, let $h=h_{2}$; otherwise let $h=h_{2}(i d \times a)$ on $E_{1}$. Thus $h=i d$ on $A_{1}$, and if the cover $\alpha$ is sufficiently fine near $Q \times$ $2 S^{m-1} \times\{0\}$, it is clear that $h$ extends to $Q \times 2 S^{m-1} \times\{0\}$ and thus to the rest of $Q \times R^{m} \times R^{n}$ by means of the identity. If $\varepsilon=r_{2}(0)$, it is trivial to check that $h$ has the required properties.

The proof of our next main lemma depends upon a similar program using pinched tubes constructed from arbitrary codimension 2 tubular neighborhoods.

While regarding tubular neighborhoods as fiber bundles, we have not emphasized the role of the structure group of the bundle and have implicitly assumed that it is the group of all homeomorphisms on $R^{n}$ which fix the origin. It follows, however, from a noted result of Kneser [14] that if an $R^{2}$-bundle admits this structure group, then it also admits as structure group the orthogonal group $O(2)$. Our use of this result is confined to noting that, for any $R^{2}$-bundle $p: E \rightarrow B$ with 0 -section, there exists a family of coordinate charts $\varphi_{i}$ such that if $x \in E$ and $\varphi_{i}(p(x), y)=x$, then $\|y\|$ depends only on $x$ and not on the particular choice of index $i$. We will denote this value by $\|x\|_{p}$.

This observation is the key to the construction of codimension 2 pinched tubes. Let $C$ be a compact $Q$-manifold, let $D$ be a tubular neighborhood of $C \times R$ with retraction $p: D \rightarrow C \times R$, and let $B=$ $C \times(-2,2)$. Then for any choice of a pinching function $r:(-2,2) \rightarrow$ $(0, \infty)$ (having the properties listed earlier), we construct a pinched tube $(E, p \mid E, B)$ by defining

$$
E=\left\{x \in D: p(x)=(y, t) \in B \text { and }\|x\|_{p} \leqq r(t)\right\} .
$$

In an analogous fashion we define the boundary of $E$ to be $A=$ $\left\{x \in E:\|x\|_{p}=r(t)\right\}$.

The following is the analogue to Proposition 4.1. 
Proposition 4.4. Let $(E, p \mid E, B)$ be a pinched tube constructed from a codimension 2 tube as described above.

(1) Given any neighborhood $U$ of $B$ in $D$, there is a choice of pinching function $r$ so that $E \subset U$.

(2) Any two such tubes, $\left(E_{1}, p \mid E_{1}, B\right)$ and $\left(E_{2}, p \mid E_{2}, B\right)$, constructed from the same fiber bundle are isomorphic $D^{2}$-bundles, and $\left(A_{1}, p \mid A_{1}, B\right)$ and $\left(A_{2}, p \mid A_{2}, B\right)$ are isomorphic $S^{1}$-bundles.

(3) There exists a fiber preserving retraction $\rho: p^{-1}(B)-B \rightarrow A$.

Conclusions (1) and (2) are immediate consequences of the definitions. Conclusion (3) can be proved by a routine Tietze extension proof using the orthogonality of the structure group. The details are left to the reader.

We will also need the corollary to the following lemma. The lemma itself is well known in bundle theory.

LeMmA 4.5. Let $B$ be a locally finite polyhedron. If $p_{1}: E_{1} \rightarrow B$ and $p_{2}: E_{2} \rightarrow B$ are $S^{1}$-bundles and if $f: E_{1} \rightarrow E_{2}$ is a fiber homotopy equivalence, then $f$ is fiber homotopic to a homeomorphism.

COROLLARY 4.6. Let $B$ be a Q-manifold. Then the conclusion of 4.6 holds.

Proof. Triangulate $B$ as $P \times Q$ where $P$ is a locally finite polyhedron. Let $\widetilde{E}_{i}=p_{i}^{-1}(P \times\{0\}), i=1,2$, and let $r: B \rightarrow P \times\{0\}$ be a deformation retraction. It follows readily from the homotopy properties of bundles (see Chapter 4 of [11], especially Theorem 9.9) that $p_{i}: E_{i} \rightarrow B$ is isomorphic to the induced bundle $r^{*}\left(p_{i}\right): r^{*}\left(\widetilde{E_{\imath}}\right) \rightarrow B$, where $r^{*}\left(\widetilde{E}_{i}\right)=\left\{(x, b) \in \widetilde{E}_{i} \times B: p_{i}(x)=r(b)\right\}$ and $r^{*}\left(p_{i}\right)(x, b)=b$. Note moreover that any fiber preserving map $g: \widetilde{E}_{1} \rightarrow \widetilde{E}_{1}$ induces a fiber preserving map $r^{*}(g): r^{*}\left(\widetilde{E}_{1}\right) \rightarrow r^{*}\left(\widetilde{E}_{1}\right)$ defined by $r^{*}(g)(x, b)=(g(x), b)$. Now apply Lemma 4.5 to $f \mid \widetilde{E}_{1}: \widetilde{E}_{1} \rightarrow \widetilde{E}_{2}$.

We are now ready to prove our second main meshing lemma.

LEMMA 4.7. Let $M$ be a compact $Q$-manifold and let $p: D \rightarrow$ $M \times R$ and $p^{\prime}: D^{\prime} \rightarrow M \times R$ be $R^{2}$-bundles such that $D^{\prime} \subset D$ and such that $M \times R \hookrightarrow D$ and $M \times R \hookrightarrow D^{\prime}$ are 0-sections. Then there exists a homeomorphism $h: D \rightarrow D$ such that

(a) h has compact support,

(b) $h=i d$ on $M \times R$, and

(c) $h$ is a microbundle isomorphism over $M \times[-1,1]$ (that is, there is a neighborhood $U$ of $M \times[-1,1]$ in $D$ such that $h(U) \subset D^{\prime}$ and $p^{\prime} h=p$ on $U$ ). 
Proof. Let $B=M \times(-2,2)$. In a manner analogous to the proof of Lemma 4.3 we construct pinched tubes $\left(E_{1}, p_{1}, B\right)$ and $\left(E_{3}, p_{3}, B\right)$ from $p: D \rightarrow M \times R$ and a pinched tube $\left(E_{2}, p_{2}, B\right)$ from $p^{\prime}: D^{\prime} \rightarrow M \times R$ such that $E_{1} \subset D^{\prime}, E_{2} \subset \dot{E}_{1}$, and $E_{3} \subset E_{2}^{\circ}$. As before, let $A_{k}$ be the boundary of $E_{k}$ and let $X=E_{1}-\dot{E}_{2}$. It is routine to verify that the $E_{k}$, the $A_{k}$ and $X$ are all $Q$-manifolds.

By Proposition 4.4 (3) there is a fiber preserving retraction $\rho:\left(p^{\prime}\right)^{-1}(B)-B \rightarrow A_{2}$. It follows from Proposition 4.4 (2) that there is a natural fiber preserving homeomorphism $\sigma: E_{1} \rightarrow E_{3}$ which preserves $B$ and maps $A_{1}$ to $A_{3}$. Moreover, it is clear that there is a fiber preserving isotopy $\sigma_{t}: E_{1} \rightarrow E_{1}$ such that $\sigma_{0}=i d$ and $\sigma_{1}=\sigma$. Let $u=\rho \sigma$. Finally, in an analogue to the proof of Lemma 4.3, define a retraction $\rho_{1}: E_{1}-B \rightarrow X$ by $\rho_{1}=\rho$ on $E_{2}-B$ and $\rho_{1}=i d$ on $E_{1}-E_{2}$.

Assertion 1. The inclusion $i: A_{2} \hookrightarrow X$ is a proper homotopy equivalence and $u: X \rightarrow A_{2}$ is a homotopy inverse for $i$. Moreover, if $\beta$ is an open cover of $B$, then the pinched tubes can be chosen so that $u$ is a $\left(p^{\prime}\right)^{-1}(\beta)$-equivalence.

Proof. The proof is the same as that for Assertion 1 in Lemma 4.3 once the appropriate functions have been defined as above.

Again we use Proposition $4.4(3)$ and let $\tau: p^{-1}(B)-B \rightarrow A_{3}$ be a fiber preserving retraction.

Assertion 2. $\tau \mid A_{2}: A_{2} \rightarrow A_{3}$ is a proper homotopy equivalence. Moreover, if $\beta$ is an open cover of $B$, then the pinched tubes can be chosen so that $\tau$ is a $p^{-1}(\beta)$-equivalence.

Proof. We show that $\rho \mid A_{3}: A_{3} \rightarrow A_{2}$ is a proper homotopy inverse. Define $\tau_{1}: E_{1}-B \rightarrow E_{1}-\dot{E}_{3}$ by $\tau_{1}=\tau$ on $E_{3}-B$ and $\tau_{1}=i d$ on $E_{1}-E_{3}$. Then $\rho \tau_{1} \sigma_{t} \mid A_{2}: A_{2} \rightarrow A_{2}$ is a proper homotopy in $A_{2}$ between $\rho \tau$ and the identity and $\tau \rho_{1} \sigma_{t}^{-1} \mid A_{3}: A_{3} \rightarrow A_{3}$ is a proper homotopy in $A_{3}$ between $\tau \rho$ and the identity. Proof of the cover control is routine.

Concider now the $S^{1}$-bundles $\left(A_{2}, p^{\prime} \mid A_{2}, B\right)$ and $\left(A_{3}, p \mid A_{3}, B\right)$.

Assertion 3. The pinched tubes can be chosen so that $\tau \mid A_{2}$ is properly homotopic to a fiber preserving homeomorphism $f: A_{2} \rightarrow A_{3}$. Moreover, if $\alpha$ is an open cover of $B$, the pinched tubes can be chosen so that the homotopy is a $p^{-1}(\alpha)$-homotopy. 
Proof. The bundle projections $p$ and $p^{\prime}$ agree on $B$ and are thus close as maps near $B$. Therefore it follows from an elementary continuity argument and well known properties of ANRs that, so long as the values of the pinching function are chosen sufficiently small, we can require that $p \stackrel{\alpha}{\cong} p^{\prime}$ on $E_{2}$. Let $F_{t}: A_{2} \rightarrow B$ be an $\alpha$ homotopy with $F_{0}=p$ and $F_{1}=p^{\prime}$. Since $p \tau=p$, by the covering homotopy property $F_{t}$ lifts to a $p^{-1}(\alpha)$-homotopy $H_{t}: A_{2} \rightarrow A_{3}$ with $H_{0}=\tau$ and $p H_{1}=F_{1}=p^{\prime}$. Since $\tau \mid A_{2}$ is a homotopy equivalence, $H_{1}$ is also a homotopy equivalence; moreover $H_{1}$ is fiber preserving. According to [6, Theorem 6.1] such a fiber preserving homotopy equivalence is in fact a fiber homotopy equivalence. Thus by Corollary 4.6, $H_{1}$ is fiber homotopic to the desired homeomorphism $f$.

Let $\tilde{f}: E_{2} \rightarrow E_{3}$ be the natural extension of $f$ from a fiber preserving homeomorphism between $S^{1}$-bundles to a fiber preserving homeomorphism between the associated $D^{2}$-bundles.

Assertion 4. If $\alpha$ is any open cover of $B$, then the pinched tubes can be chosen so that there is a homeomorphism $h_{1}: E_{1} \rightarrow E_{1}$ such that

(1) $h_{1} \mid A_{1}: A_{1} \rightarrow A_{1}$ is fiber preserving homeomorphism,

(2) $h_{1}=\tilde{f}$ on $E_{2}$, and

(3) $h_{1}$ is $p^{-1}(\alpha)$-close to the identity.

Proof. The proof is virtually the same as the proof of Assertion 2 in Lemma 4.3, so we indicate only the necessary modifications.

In order to obtain conditions (2) and (3), the only modifications are to replace, of course, $f^{-1}$ with $\tilde{f}$ and to redefine $\psi$ to be the natural homeomorphism $\psi: A_{3} \times I \rightarrow E_{1}-\check{E}_{3}$.

In order to obtain condition (1), we incorporate the following into the $Z$-set unknotting. We choose as before the homeomorphism $\bar{g}: X \rightarrow A_{2} \times I$ so that $\bar{g} \mid A_{2} \simeq u \times 0$ and $\bar{g} \mid A_{1} \simeq u \times 1$. Now since $\tau \simeq \widetilde{f}$ on $A_{2}, \tilde{f}^{-1} \tau \simeq i d$ on $A_{2}$. Furthermore, recall that $\sigma\left(A_{1}\right)=A_{3}$, where $\sigma$ is the previously defined fiber preserving homeomorphism, and that $\tau \rho \simeq i d$ on $A_{3}$. Therefore

$$
\bar{g} \mid A_{1} \simeq \rho \sigma \times 1 \simeq \tilde{f}^{-1} \tau \rho \sigma \times 1 \simeq \tilde{f}^{-1} \sigma \times 1 .
$$

Moreover, all of these homotopies have been shown to have arbitrarily fine horizontal control. Therefore, the controlled $Z$-set unknotting yields a modified $\bar{g}$ so that $\bar{g} \mid A_{2}=i d \times 0$ and $\bar{g} \mid A_{1}=\tilde{f}^{-1} \sigma \times 1$. All other details are as before.

Now we complete the proof of the lemma. Let $h_{2}: E_{1} \rightarrow E_{1}$ be the natural fiber preserving (with respect to $p_{1}$ ) extension of $h_{1} \mid A_{1}$. 
Define $h: E \rightarrow E$ by $h=h_{1}^{-1} h_{2}$. Then $h=i d$ on $A_{1}$ and $p h$ is arbitrarily close to $p$. Thus $h$ can be extended continuously by the identity to all of $D$. It is easy to verify that $h$ is the desired homeomorphism.

We conclude this section with the statement of an alternate version of the second meshing lemma. This version will be used in proving the uniqueness part of Theorem 2. The proof, including the preliminary constructions, is the same as the proof of Lemma 4.7 with the following exception. The base space for the pinched tubes is taken to be an arbitrary $Q$-manifold. Consequently, the closure of the pinched tubes in the total space of the given bundle need not be compact, so the conclusion that $h$ have compact support is removed.

Lemma 4.8. Let $B$ be a Q-manifold and let $p_{k}$ : $E_{k} \rightarrow B, k=1,2$, be $R^{2}$-bundles such that $E_{2} \subset E_{1}$ and such that $B \hookrightarrow E_{k}$ is a 0-section. Let $\alpha$ be an open cover of $B$ and let $U$ be a neighborhood of $B$ in $E_{1}$. Then there exists a homeomorphism $h: E_{1} \rightarrow E_{1}$ such that

(a) $h$ is supported in $U$,

(b) $h$ is $p_{1}^{-1}(\alpha)$-close to the identity,

(c) $h=i d$ on $B$, and

(d) $h$ is a microbundle isomorphism over $B$.

5. Proof of Theorem 1. Let $i: Q \times R^{m} \rightarrow M$ be a locally flat embedding. Since $N=i\left(Q \times R^{m}\right)$ is homeomorphic to $Q \times R^{m} \times I$, we simplify notation by identifying $N$ and $Q \times R^{m} \times I$.

Since the inclusion $N \hookrightarrow M$ is locally flat, there exists a neighborhood $U$ of $(0,0,0) \in N$ and an open embedding $\varphi: U \times R^{n} \rightarrow M$ such that $\varphi \in i d$ on $U \times\{0\}$. The first step in the proof is to enlarge $U$ so as to engulf $Q \times B^{m} \times\{0\}$.

Since $\{(0,0,0)\}$ is a $Z$-set in $N$, we can use 2.2 to conclude that there is a $Z$-embedding $f: Q \times B^{m} \times\{0\} \rightarrow N$ such that $f(0,0,0)=$ $(0,0,0)$ and $f\left(Q \times B^{m} \times\{0\}\right) \subset U$. Since $Q \times B^{m}$ is contractible, both $f$ and the inclusion $Q \times B^{m} \times\{0\} \hookrightarrow N$ are proper homotopic to the constant map to $(0,0,0)$. Thus it follows from relative $Z$-set unknotting (Theorem 3.2) that there exists a homeomorphism $g$ : $(M, N) \rightarrow(M, N)$ such that $g=f$ on $Q \times B^{m} \times\{0\}$.

Then $\widetilde{U}=g^{-1}(U)$ is an open neighborhood of $Q \times B^{m} \times\{0\}$, and an open embedding $\widetilde{\varphi}: \widetilde{U} \times R^{n} \rightarrow M$ can be defined by $\widetilde{\varphi}(x, y)=$ $g^{-1} \varphi(g(x), y)$. Note further that $\widetilde{\varphi}=i d$ on $\widetilde{U} \times\{0\}$.

Our next step is to show that the embedding is flat over a neighborhood of $Q \times\{0\} \times I$ in $N$. Choose $\varepsilon>0$ so that $Q \times B^{m} \times$ $[0, \varepsilon] \subset \widetilde{U}$. By Lemma 3.1 there is a homeomorphism $h:(M, N) \rightarrow$ 
$(M, N)$ such that $h(x, \varepsilon)=(x, 3 / 4)$ for each $x \in Q \times R^{m}$. Define an open embedding

$$
\psi_{1}: Q \times \stackrel{\circ}{B}^{m} \times\left[0, \frac{3}{4}\right) \times R^{n} \longrightarrow M
$$

by

$$
\psi_{1}(x, t, y)=h \widetilde{\varphi}\left(h^{-1}(x, t), y\right) .
$$

Note that $\psi_{1}(x, t, 0)=(x, t, 0)$. Now a similar construction gives us an open embedding

$$
\psi_{2}: Q \times \stackrel{\circ}{B}^{m} \times\left(\frac{1}{4}, 1\right] \times R^{n} \longrightarrow M
$$

such that $\psi_{2}(x, t, 0)=(x, t, 0)$. Here is a picture illustrating the idea.

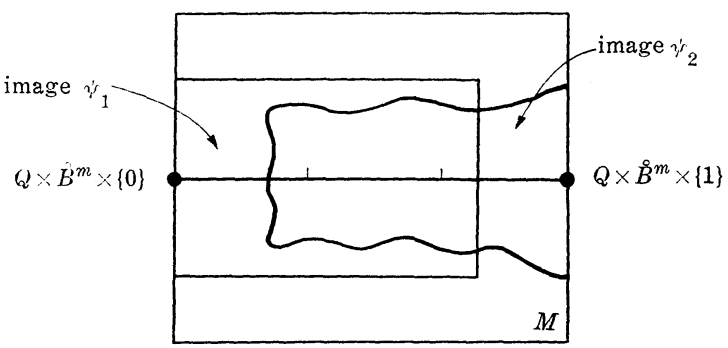

We will use Lemma 4.3 to piece these embeddings together.

Without loss of generality we can require that

$$
\psi_{2}\left(Q \times \stackrel{\circ}{B}^{m} \times\left[\frac{1}{3}, \frac{2}{3}\right] \times R^{n}\right) \subset \psi_{1}\left(Q \times \stackrel{\circ}{B}^{m} \times\left(\frac{1}{4}, \frac{3}{4}\right) \times R^{n}\right) .
$$

It is then easy to show that there is an open embedding

$$
\sigma: \psi_{2}\left(Q \times \stackrel{\circ}{B}^{m} \times\left(\frac{1}{4}, \frac{3}{4}\right) \times R^{n}\right) \longrightarrow \psi_{1}\left(Q \times \stackrel{\circ}{B}^{m} \times\left(\frac{1}{4}, \frac{3}{4}\right) \times R^{n}\right)
$$

such that $\sigma=i d$ on $Q \times \dot{B}^{m} \times(1 / 4,3 / 4) \times\{0\}$ and on $\psi_{2}\left(Q \times \stackrel{\circ}{B}^{m} \times\right.$ $\left.[1 / 3,2 / 3] \times R^{n}\right)$. Therefore we define $\theta=\psi_{1}^{-1} \sigma \psi_{2}$ and obtain an open embedding

$$
\theta: Q \times \dot{B}^{m} \times\left(\frac{1}{4}, \frac{3}{4}\right) \times R^{n} \longrightarrow Q \times \dot{B}^{m} \times\left(\frac{1}{4}, \frac{3}{4}\right) \times R^{n}
$$

such that $\theta=i d$ on $Q \times B^{m} \times(1 / 4,3 / 4) \times\{0\}$ and $\theta=\psi_{1}^{-1} \psi_{2}$ on $Q \times$ $\stackrel{\circ}{B}^{m} \times[1 / 3,2 / 3] \times R^{n}$. Since $\left(\stackrel{\circ}{ }^{m} \times(1 / 4,3 / 4), 1 / 2 B^{m} \times[1 / 3,2 / 3]\right)$ is naturally homeomorphic to $\left(R^{m+1}, B^{m+1}\right)$, it follows from Lemma 4.3 that there is a homeomorphism 


$$
h: Q \times \dot{B}^{m} \times\left(\frac{1}{4}, \frac{3}{4}\right) \times R^{n} \longrightarrow Q \times \dot{\circ}^{m} \times\left(\frac{1}{4}, \frac{3}{4}\right) \times R^{n}
$$

such that

(1) $h$ has compact support,

(2) $h=i d$ on $Q \times \stackrel{B}{m}^{m} \times(1 / 4,3 / 4) \times\{0\}$, and

(3) for some choice of $\varepsilon, 0<\varepsilon<1$, either $h \theta=i d$ or $h \theta=i d \times a$ on $Q \times 1 / 2 B^{m} \times[1 / 3,2 / 3] \times \varepsilon B^{n}$.

Define a homeomorphism $\tilde{h}: M \rightarrow M$ by $\tilde{h}=\psi_{1} h \psi_{1}^{-1}$ on $\psi_{1}\left(Q \times B^{m} \times\right.$ $\left.(1 / 4,3 / 4) \times R^{n}\right)$ and $\widetilde{h}=i d$ elsewhere.

Define an embedding

$$
\psi_{2}: Q \times \dot{B}^{m} \times\left(\frac{1}{4}, 1\right] \times R^{n} \longrightarrow M
$$

as follows. In case $h \theta=i d$ on $Q \times 1 / 2 B^{m} \times[1 / 3,2 / 3] \times \varepsilon B^{n}$, let $\widetilde{\psi}_{2}=$ $\widetilde{h} \psi_{2}$. In case $h \theta=i d \times a$ when so restricted, let $\widetilde{\psi}_{2}=\tilde{h} \psi_{2}(i d \times a)$. Let $\rho: R^{n} \rightarrow \varepsilon B^{n}$ be a radially defined homeomorphism. We can therefore define an open embedding

$$
\eta: Q \times \frac{1}{2} \dot{B}^{m} \times[0,1] \times R^{n} \longrightarrow M
$$

by $\eta(x, t, y)=\psi_{1}(x, t, \rho(y))$ if $0 \leqq t \leqq 2 / 3$ and $\eta(x, t, y)=\widetilde{\psi}_{2}(x, t, \rho(y))$ if $1 / 3 \leqq t \leqq 1$. The construction guarantees that $\eta$ is well defined and that $\eta=i d$ on $Q \times 1 / 2 B^{m} \times[0,1] \times\{0\}$. Also, without loss of generality we may assume that $\eta(x, t, y) \notin N$ if $y \neq 0$.

This completes the second step in the proof.

The proof will be complete once we extend the product embedding over the $R^{m}$ factor. This is obtained by a sequence of engulfings.

Choose a sequence $\left\{r_{j}\right\}$ of positive real numbers such that $1 / 4<$ $r_{1}<\cdots<r_{j} \cdots$ and $\lim _{j \rightarrow \infty} r_{j}=1 / 2$. A piecewise linear homeomorphism $f_{1}:[1 / 4,2] \rightarrow[0,1]$ such that $f_{1}\left(r_{1}\right)=1 / 3$ and $f_{1}(1)=2 / 3$ induces an obvious homeomorphism

$$
g_{1}: Q \times\left(2 B^{m}-\frac{1}{4} \stackrel{\circ}{B}^{m}\right) \times I \longrightarrow\left(Q \times S^{m-1} \times I\right) \times[0,1] .
$$

Let $U_{1}$ be an open set of $M$ such that

$$
Q \times\left(B^{m}-r_{1} \dot{B}^{m}\right) \times I \subset U_{1} \cap N \subset Q \times\left(2 B^{m}-\frac{1}{4} \stackrel{\circ}{B}^{m}\right) .
$$

By Lemma 3.1 there is a homeomorphism

$$
h_{1}:\left(M, Q \times\left(2 B^{m}-\frac{1}{4} \stackrel{\circ}{ }^{m}\right) \times I\right) \longrightarrow\left(M, Q \times\left(2 B^{m}-\frac{1}{4} \stackrel{\circ}{B}^{m}\right) \times I\right)
$$


whose support is a compact subset of $U_{1}$ and such that $h_{1} g_{1}^{-1}(x, 1 / 3)=$ $g_{1}^{-1}(x, 2 / 3)$. Thus

$$
h_{1}\left(Q \times r_{1} S^{m-1} \times I\right)=Q \times S^{m-1} \times I .
$$

Clearly we also have $h_{1}\left(Q \times r_{2} S^{m-1} \times I\right) \subset Q \times\left(2 \dot{B}^{m}-B^{m}\right) \times I$. Thus we can proceed in like manner to define a sequence of homeomorphisms

$$
\begin{aligned}
h_{j}:(M, Q & \left.\times\left((j+1) B^{m}-(j-1) B^{m}\right) \times I\right) \\
\quad \longrightarrow & \left(M, Q \times\left((j+1) B^{m}-(j-1) B^{m}\right) \times I\right), \quad j=2,3, \cdots,
\end{aligned}
$$

such that the following conditions are satisfied.

(1) The support of $h_{j}$ is a compact subset of an open set $U_{j}$ of $M$ such that the intersection of $U_{j}$ and the support of $h_{k}$ is empty whenever $j \geqq 3$ and $k \leqq j-2$.

(2) $\quad h_{j} h_{j-1} \cdots h_{1}\left(Q \times r_{j} S^{m-1} \times I\right)=Q \times j S^{k-1} \times I$.

Now define $h:\left(M, Q \times 1 / 2 \dot{B}^{m} \times I\right) \rightarrow(M, N)$ by $h(x)=\lim _{j \rightarrow \infty} h_{j} \cdots h_{1}(x)$. The supports of the $h_{j}$ were chosen so that for each $x \in M$, there exists some integer $m$ such that $h(y)=h_{m} \cdots h_{1}(y)$ for all $y$ in a neighborhood of $x$. Therefore $h$ is a well defined homeomorphism. Property (2) above and the choice of the $r_{j}$ guarantee that $h \mid Q \times$ $1 / 2 B^{m} \times I$ is a homeomorphism onto $N$.

Therefore the desired open embedding $\gamma: N \times R^{n} \rightarrow M$ is defined by $\gamma(x, y)=h \eta\left(h^{-1}(x), y\right)$.

6. Proof of Theorem 2. The proof of Theorem 2 is broken into three parts. Theorem 6.1 is a special case of the existence portion of the theorem, Theorem 6.2 is the general existence theorem, and Theorem 6.4 is the uniqueness part of the theorem.

Theorem 6.1. Let $M$ be a Q-manifold and let $K$ be a finite dimensional polyhedron. If $i: K \times Q \rightarrow M$ is a locally flat embedding of codimension 2, then $N=i(K \times Q)$ has a tubular neighborhood in $M$.

Proof. We identify $N$ with $K \times Q$ and argue inductively on the dimension of $K$.

In case $\operatorname{dim} K=0, N$ is the disjoint union of copies of $Q$. Thus each component of $N$ consists of a locally flat embedding of $Q$ in $M$, which, by Theorem 1, is flat. That is, each component of $N$ has a neighborhood in $M$ homeomorphic to $Q \times R^{2}$, and, since it is clear that these neighborhoods can be chosen to be disjoint, the inclusion 
$N \hookrightarrow M$ is therefore flat. This disposes of the case for which $\operatorname{dim} K=0$.

For the inductive step, assume the theorem to be true for any polyhedron $L$ such that $\operatorname{dim} L=n$ and consider a specific case in which $\operatorname{dim} K=n+1$. Without loss of generality we assume $K=$ $K^{n} \cup \Delta^{n+1}$ where $K^{n}$ is the space of the $n$-skeleton of $K$ and $\Delta^{n+1}$ is a single $(n+1)$-simplex.

Let $A$ be the interior of $A^{n+1}$ and let $M_{1}$ be any open submanifold of $M$ so that $A \times Q$ is closed in $M_{1}$. Since the inclusion $K \times Q \hookrightarrow M$ is locally flat, it is clear that the inclusion $A \times Q \hookrightarrow M_{1}$ is locally flat, and, since $A$ is homeomorphic to $R^{n+1}$, by Theorem 1 the inclusion is flat. That is, there is an open embedding $\varphi:(A \times Q) \times R^{2} \rightarrow$ $M_{1} \subset M$ such that $\varphi(x, 0)=x$.

Now identify $\Delta^{n+1}$ with $B^{n+1} \cup\left(S^{n} \times I\right)$ so that $A$ is identified with $B^{n+1} \cup\left(S^{n} \times[0,1)\right)$. Let $L=K^{n} \cup\left(S^{n} \times[0,1]\right)$. It is geometrically obvious that $K^{n}$ expands to $L$ by an elementary expansion so the inclusion $K^{n} \hookrightarrow L$ is a simple homotopy equivalence. (See Chapter VIII of [2] for an explanation of the terminology.) Therefore, by a theorem of West [2, Theorem 29.4], $L \times Q$ is homeomorphic to $K^{n} \times Q$. Let $M_{2}=M-\varphi\left(\dot{B}^{n+1} \times Q \times \dot{B}^{2}\right)$. The trivial tube structure implies that $M_{2}$ is a $Q$-manifold. Moreover, the inclusion $L \times Q \hookrightarrow M_{2}$ is locally flat, so by the induction hypothesis there is a tubular neighborhood $E$ of $L \times Q$ in $M_{2}$. Let $p: E \rightarrow L \times Q$ be the associated retraction.

We wish to use our second meshing lemma to mesh the trivial tube over $A$ with $E$. Let $B=S^{n} \times(0,1) \times Q \subset(A \times Q) \cap(L \times Q)$. Since

$$
B \subset p^{-1}(B) \cap \varphi\left(B \times R^{2}\right) \stackrel{p \mid}{\longrightarrow} B
$$

is isomorphic as a normal microbundle to the restriction of $p^{-1}(B)$, we may assume without loss of generality that $p^{-1}(B) \subset \varphi\left(B \times R^{2}\right)$. Then by Lemma 4.7 there is a homeomorphism $h: \varphi\left(B \times R^{2}\right) \rightarrow$ $\varphi\left(B \times R^{2}\right)$ such that

(1) $h$ has compact support,

(2) $h=i d$ on $B$, and

(3) there is a neighborhood $U$ of $\left(S^{n} \times[1 / 3,2 / 3]\right) \times Q \subset B$ such that if $\varphi(x, y) \in U$, then $p h \varphi(x, y)=x$.

Because of (1) $h$ extends by the identity to all of $M$. Choose $\varepsilon>0$ so that

$$
\varphi\left(S^{n} \times\left[\frac{1}{3}, \frac{2}{3}\right] \times Q \times \varepsilon B^{2}\right) \subset U,
$$

and let $\gamma: R^{2} \rightarrow \varepsilon \dot{B}^{2}$ be a radially defined homeomorphism. Then 


$$
\widetilde{\varphi}=h \varphi(i d \times \gamma):(A \times Q) \times R^{2} \longrightarrow M
$$

is an open embedding. Let $\widetilde{E}=E_{1} \cup E_{2}$ where

$$
\begin{aligned}
& E_{1}=p^{-1}\left(\left(K^{n} \cup\left(S^{n} \times\left(\frac{1}{3}, 1\right)\right)\right) \times Q\right) \text { and } \\
& E_{2}=\widetilde{\Phi}\left(\left(B^{n+1} \cup\left(S^{n} \times\left(0, \frac{2}{3}\right)\right)\right) \times Q \times R^{2}\right)
\end{aligned}
$$

and define $\widetilde{p}: \widetilde{E} \rightarrow K \times Q$ by

$$
\begin{aligned}
& \widetilde{p}=p \text { on } E_{1} \text { and } \\
& \widetilde{p}(\widetilde{\varphi}(x, q, y))=(x, q) \text { if } \widetilde{\varphi}(x, q, y) \in E_{2} .
\end{aligned}
$$

Here is a picture.

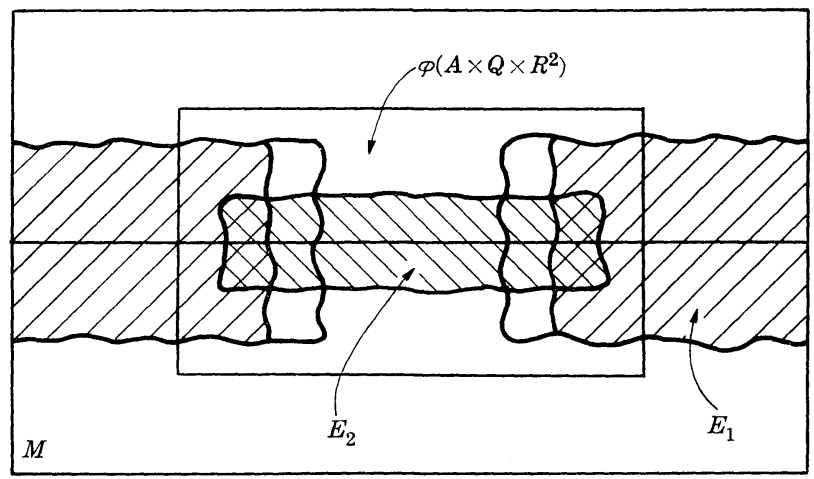

It is easy to check that $\widetilde{p}$ is well defined and that $K \times Q \hookrightarrow \widetilde{E} \stackrel{\tilde{p}}{\rightarrow}$ $K \times Q$ is a normal microbundle. Therefore, by the Kister-Mazur theorem, $\widetilde{E}$ contains a tubular neighborbood of $K \times Q$.

THEOREM 6.2. Let $(M, N)$ be a Q-manifold pair. If $N \hookrightarrow M$ is a locally flat embedding of codimension 2, then $N$ a tubular neighborhood in $M$.

Proof. Triangulate $N$ as $K \times Q$ where $K$ is a locally finite polyhedron. The following decomposition of $K$ is routine.

There exists a sequence $A_{1}, A_{2}, \cdots$ of open subsets of $K$ such that

(1) $K=\bigcup_{i=1}^{\infty} A_{i}$

(2) for each $i, \bar{A}_{i}$ is a compact subset of $A_{i+1}$, and

(3) the boundary of $A_{i}$ is collared in $A_{i+1}-A_{i}$; that is, there is an open embedding $u_{i}: \mathrm{Bd} A_{i} \times[0,1) \rightarrow A_{i+1}-A_{i}$ so that $u_{i}(x, 0)=x$ for each $x \in A_{i}$.

Now define $B_{i}=\left(A_{i}-\bar{A}_{i-1}\right) \cup u_{i}\left(\mathrm{Bd} A_{i} \times[0,1)\right)$. It clearly follows 
from Theorem 6.1 that each $B_{i} \times Q$ has a tubular neighborhood $E_{i}$ in $M$.

For each $i, E_{i}$ and $E_{i+1}$ can be meshed to form a normal microbundle for $\left(B_{i} \cup B_{i+1}\right) \times Q$ by a procedure similar to that used in the proof of Theorem 6.1. Moreover, it is clear that the meshing homeomorphisms can be chosen so as to have disjoint supports. Therefore, the $E_{i}$ can be meshed to form a normal microbundle and hence a tubular neighborhood for all of $K \times Q$.

In order to prove uniqueness we need the following relative version of the local contractibility of the homeomorphism group of a $Q$-manifold. It follows from the Fathi-Visetti deformation theorem [8] in the same way that the corresponding finite dimensional result follows from the Edwards-Kirby deformation theorem (see [7, Remark 7.2]).

Lemma 6.3. Let $(M, N)$ be a Q-manifold pair such that $N \hookrightarrow M$ is locally flat. If $h: M \rightarrow M$ is a homeomorphism such that $h=i d$ on $N$ and if $h$ is sufficiently close to id $d_{M}$ then there exists an isotopy $h_{t}: h \simeq i d$ such that $h_{t}=i d$ on $N$ for all $t \in I$.

TheOREM 6.4. Let $(M, N)$ be a Q-manifold pair such that $N$ has a codimension 2 tubular neighborhood. Then any two normal microbundles of $N$ are isotopic.

Proof. Let $p_{1}: E_{1} \rightarrow N$ and $p_{2}: E_{2} \rightarrow N$ determine tubular neighborhoods for $N$. Lemma 4.8 provides a microbundle isomorphism $h$ between the tubes. We wish to use Lemma 6.3 to conclude that $h$ is isotopic to the identity modulo $N$. It is thus necessary to show that $h$ can be chosen orbitrarily close to the identity.

Since $N$ has neighborhoods which are locally products of open sets of $N$ with $R^{2}$, a map is close to the identity near $N$ if it is close to the identity with respect to this product structure. A map is in turn close to the identity in the $R^{2}$ factor if it is the identity at the origin and is supported on a sufficiently small neighborhood of the origin. The following is the precise statement we need. Its proof is a routine continuity argument.

Let $\alpha$ be an open cover of $M$. Then there exists a neighborhood $U$ of $N$ in $M$ and there exists an open cover $\beta$ of $N$ so that a map $f: M \rightarrow M$ is $\alpha$-close to $i d$ whenever the following conditions are satisfied.

(1) $f=i d$ on $N$.

(2) $f$ is supported on $U$. 
(3) $p_{1} f$ is $\beta$-close $p_{1}$ on $E_{1}$. But this is exactly the control available from Lemma 4.8. The result therefore follows immediately from a combination of Lemma 4.8 and Lemma 6.3.

\section{REFERENCES}

1. M. Brown, Locally flat embeddings of topological manifolds, Annals of Math., 75 (1962), 331-342.

2. T. A. Chapman, Lectures on Hilbert cube manifolds, C. B. M. S. Regional Conference Series in Math., No. 28, 1976.

3. - Locally flat embeddings of Hilbert cubes are flat, Fundamenta Math., 87 (1975), 184-193.

4. - Constructing locally flat embeddings of infinite dimensional manifolds without tubular neighborhoods, preprint.

5. - Approximating maps into fiber bundles by homeomorphisms, preprint.

6. A. Dold, Partitions of unity in the theory of fibrations, Annals of Math., 78 (1963), 223-255.

7. R. D. Edwards and R. C. Kirby, Deformations of spaces of embeddings, Annals of Math., 93 (1971), 63-88.

8. A. Fathi and Y. M. Visetti, Deformations of open embeddings of Q-manifolds, Trans. Amer. Math. Soc., 224 (1976), 427-435.

9. M. W. Hirsch, On tubular neighborhoods of piecewise linear and topological manifolds, Conference on the Topology of Manifolds, Prindle, Boston, 1968.

10. Per Holm, The microbundle representation theorem, Acta Math., 117 (1967), 191-213.

11. Dale Husemoller, Fibre Bundles, McGraw-Hill, 1966.

12. R. C. Kirby and L. C. Siebenmann, Normal bundles for codimension 2 locally flat embeddings, Proc. Topology Conference, Park City, Utah, Feb. 1974, Springer Lecture Notes in Math. 438, 1975.

13. J. M. Kister, Microbundles are fibre bundles, Annals of Math., 80 (1964), 190-199.

14. H. Kneser, Die Deformationssätze der einfach zusammenhägenden Flachen, Math.

Z., 25 (1926), 362-372.

15. N. Kuiper and R. Lashof, Microbundles I, Inventiones Math., 1 (1966), 1-17.

16. J. Milnor, Microbundles I, Topology, 3, Suppl. 1. (1964), 53-80.

17. - Topological manifolds and smooth manifolds, Proc. International Conference Math., Stockholm, 1962.

18. C. P. Rourke and B. J. Sanderson, An embedding without a normal microbundle, Inventiones Math., 3 (1967), 293-299.

19. Ronald J. Stern, On topological and piecewise linear vector fields, Topology, 14 (1975), 257-269.

Received March 3, 1978 and in revised form July 5, 1978.

UNIVERSITY OF KENTUCKY

LEXINGTON, KY 40506

Current address: Texas Tech University

Lubbock, TX 79409 


\section{PACIFIC JOURNAL OF MATHEMATICS}

EDITORS

DONALD BABBITT (Managing Editor)

University of California

Los Angeles, California 90024

Hugo RossI

University of Utah

Salt Lake City, UT 84112

C. C. MOORE and ANDREW OGG

University of California

Berkeley, CA 94720

\section{J. DUGUNDJI}

Department of Mathematics University of Southern California Los Angeles, California 90007

R. Finn and J. Milgram Stanford University

Stanford, California 94305

\section{ASSOCIATE EDITORS}
E. F. BECKENBACH
B. H. Neumann
F. WOLF
K. YoSHIDA

\section{SUPPORTING INSTITUTIONS}

UNIVERSITY OF BRITISH COLUMBIA

CALIFORNIA INSTITUTE OF TECHNOLOGY

UNIVERSITY OF CALIFORNIA

MONTANA STATE UNIVERSITY

UNIVERSITY OF NEVADA, RENO

NEW MEXICO STATE UNIVERSITY

OREGON STATE UNIVERSITY

UNIVERSITY OF OREGON
UNIVERSITY OF SOUTHERN CALIFORNIA

STANFORD UNIVERSITY

UNIVERSITY OF HAWAII

UNIVERSITY OF TOKYO

UNIVERSITY OF UTAH

WASHINGTON STATE UNIVERSITY

UNIVERSITY OF WASHINGTON 


\section{Pacific Journal of Mathematics}

\section{Vol. 83, No. 1 \\ March, 1979}

Richard Neal Ball, Topological lattice-ordered groups ............... 1

Stephen Berman, On the low-dimensional cohomology of some

infinite-dimensional simple Lie algebras .................. 27

R. P. Boas and Gerald Thomas Cargo, Level sets of derivatives ......... 37

James K. Deveney and John Nelson Mordeson, Splitting and modularly

perfect fields......................................

Robert Hugh Gilman and Ronald Mark Solomon, Finite groups with small

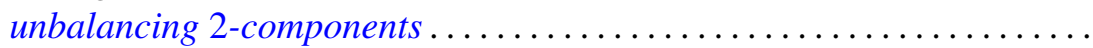

George Grätzer, Andras Hajnal and David C. Kelly, Chain conditions in free products of lattices with infinitary operations..................

Benjamin Rigler Halpern, Periodic points on tori ..................

Dean G. Hoffman and David Anthony Klarner, Sets of integers closed under

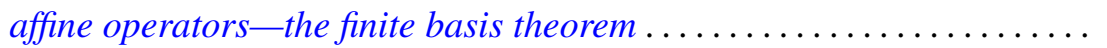

Rudolf-Eberhard Hoffmann, On the sobrification remainder ${ }^{s} X-X \ldots \ldots$

Gerald William Johnson and David Lee Skoug, Scale-invariant

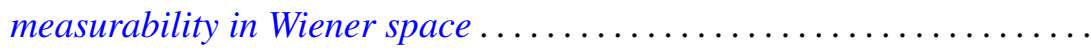

Michael Keisler, Integral representation for elements of the dual of

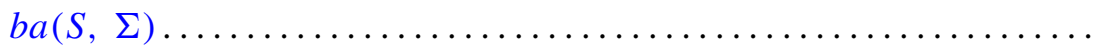

Wayne C. Bell and Michael Keisler, A characterization of the representable Lebesgue decomposition projections ................

Wadi Mahfoud, Comparison theorems for delay differential equations ...

R. Daniel Mauldin, The set of continuous nowhere differentiable functions .

Robert Wilmer Miller and Mark Lawrence Teply, The descending chain condition relative to a torsion theory...

Yoshiomi Nakagami and Colin Eric Sutherland, Takesaki's duality for regular extensions of von Neumann algebras ........ .

William Otis Nowell, Tubular neighborhoods of Hilbert cube manifolds ...

Mohan S. Putcha, Generalization of Lentin's theory of principal solutions of word equations in free semigroups to free product of copies of positive reals under addition

Amitai Regev, A primeness property for central polynomials . ...

Saburou Saitoh, The Rudin kernels on an arbitrary domain. . .

Heinrich Steinlein, Some abstract generalizations of the

Ljusternik-Schnirelmann-Borsuk covering theorem . . . 